\title{
EIGENVALUE PROBLEMS FOR $p$-LAPLACIAN DYNAMIC EQUATIONS ON TIME SCALES
}

\author{
Mingzhou Guo And Hong-Rui Sun
}

\begin{abstract}
In this paper, we are concerned with the following eigenvalue problems of $m$-point boundary value problem for $p$-Laplacian dynamic equation on time scales

$$
\begin{aligned}
& \left(\varphi_{p}\left(u^{\Delta}(t)\right)\right)^{\nabla}+\lambda h(t) f(u(t))=0, t \in(0, T), \\
& u(0)=0, \varphi_{p}\left(u^{\Delta}(T)\right)=\sum_{i=1}^{m-2} a_{i} \varphi_{p}\left(u^{\Delta}\left(\xi_{i}\right)\right),
\end{aligned}
$$

where $\varphi_{p}(u)=|u|^{p-2} u, p>1$ and $\lambda>0$ is a real parameter. Under certain assumptions, some new results on existence of one or two positive solution and nonexistence are obtained for $\lambda$ evaluated in different intervals. Our work develop and improve many known results in the literature even for the continual case. In doing so the usual restriction that $f_{0}=\lim _{u \rightarrow 0^{+}} f(u) / \varphi_{p}(u)$ and $f_{\infty}=\lim _{u \rightarrow \infty} f(u) / \varphi_{p}(u)$ exist is removed. As an applications, an example is given to illustrate the main results obtained.
\end{abstract}

\section{Introduction}

The study of dynamic equations on time scales goes back to its founder Stefan Hilger [10], and is a new area of still fairly theoretical exploration in mathematics. Motivating the subject is the notion that dynamic equations on time scales can build bridges between continuous and discrete mathematics. Further, the study of time scales has led to several important applications, e.g., in the study of insect population models, phytoremediation of metals, wound healing, economic, and epidemic models $[4,6,11,14,19]$. Throughout this work we assume a working knowledge of time scales and time scales notation, where any nonempty closed subset of $\mathbb{R}$ can serve as a time scale $\mathbb{T}$, see Hilger [10], Bohner and Peterson [5, 6].

Received August 19, 2008; Revised November 20, 2008.

2000 Mathematics Subject Classification. 34B15, 39A10.

Key words and phrases. eigenvalue, time scale, positive solution, fixed point.

Supported by the NNSF of China (10801065) and NSF of Gansu Province of China (0803RJZA096). 
Very recently, there is an increasing attention paid to question of positive solution for second order boundary value problems on time scales $[2,6,7,8$, $12,15,16]$. But very little work has been done to the existence of positive solutions for $p$-Laplacian boundary value problem on time scales $[3,17,18]$. In particular, we would like to mention some results of Anderson, Avery, and Henderson [3], Sun and Li [18], Agarwal and Lü [1], which motivate us to consider our problem.

For convenience, throughout this paper we denote $\varphi_{p}(u)$ as the $p$-Laplacian operator, i.e., $\varphi_{p}(u)=|u|^{p-2} u$ for $p>1$ with $\left(\varphi_{p}\right)^{-1}=\varphi_{q}$, where $1 / p+1 / q=1$.

In [3], Anderson, Avery, and Henderson considered the following problem

$$
\begin{gathered}
\left(\varphi_{p}\left(u^{\Delta}(t)\right)\right)^{\nabla}+c(t) f(u(t))=0, t \in(a, b), \\
u(a)-B_{0}\left(u^{\Delta}(\nu)\right)=0, u^{\Delta}(b)=0,
\end{gathered}
$$

where $\nu \in(a, b), f \in C_{l d}([0, \infty),[0, \infty)), c \in C_{l d}((a, b),[0, \infty))$ and $K_{m} x \leq$ $B_{0}(x) \leq K_{M} x$ for some positive constants $K_{m}, K_{M}$. They established the existence result of at least one positive solution by a fixed point theorem of cone expansion and compression of functional type.

In [18], Sun and Li considered the existence of at least twin or triple positive solutions to the following $p$-Laplacian $m$-point boundary value problem

$$
\begin{aligned}
& \left(\varphi_{p}\left(u^{\Delta}(t)\right)\right)^{\nabla}+a(t) f(t, u(t))=0, t \in(0, T), \\
& u(0)=0, \varphi_{p}\left(u^{\Delta}(T)\right)=\sum_{i=1}^{m-2} a_{i} \varphi_{p}\left(u^{\Delta}\left(\xi_{i}\right)\right) .
\end{aligned}
$$

The main tools used in [18] are fixed point theorems in cones.

In [1], Agarwal and Lü considered the following problem

$$
\begin{gathered}
\left(\varphi_{p}\left(u^{\prime}(t)\right)\right)^{\prime}+\lambda F(t, u(t))=0, t \in(0,1), \\
u(0)=u(1)=0,
\end{gathered}
$$

where $\alpha(t) f(u) \leq F(t, u) \leq \beta(t) f(u)$ for $t \in(0,1)$ and $u \in(0, \infty)$. They obtained series results of the existence and nonexistence of positive solution. It is noted that in the paper [1], the authors assumed that $f_{0}=\lim _{u \rightarrow 0^{+}} f(u) / \varphi_{p}(u)$ and $f_{\infty}=\lim _{x \rightarrow \infty} f(u) / \varphi_{p}(u)$ exist. However, there is little work has been done to the eigenvalue problem of multi-point boundary value problems for $p$-Laplacian dynamic equation on time scales.

In this paper we consider the eigenvalue problem of $m$-point boundary value problem for the one-dimensional $p$-Laplacian dynamic equation on time scales

$$
\begin{aligned}
& \left(\varphi_{p}\left(u^{\Delta}(t)\right)\right)^{\nabla}+\lambda h(t) f(u(t))=0, t \in(0, T), \\
& u(0)=0, \varphi_{p}\left(u^{\Delta}(T)\right)=\sum_{i=1}^{m-2} a_{i} \varphi_{p}\left(u^{\Delta}\left(\xi_{i}\right)\right) .
\end{aligned}
$$


Under certain assumptions, results on existence of one or two positive solution and nonexistence are obtained for $\lambda$ evaluated in different intervals. In doing so the usual restriction that $f_{0}=\lim _{u \rightarrow 0^{+}} f(u) / \varphi_{p}(u)$ and $f_{\infty}=$ $\lim _{x \rightarrow \infty} f(u) / \varphi_{p}(u)$ exist is removed. The results are even new for the special cases of difference equations and differential equations, as well as in the general time scale setting.

The rest of this paper is organized as follows. In Section 2, we shall provide some preliminaries. For convenience, we also state the Krasnosel'skii's fixed point theorem in a cone. In Section 3, we characterize the value of $\lambda$ so that (1.1), (1.2) has a positive solution. Section 4 is due to develop existence criteria of single and twin positive solutions for any $\lambda$ in the interval which we have given in Section 2. In the last section, we will consider the conditions of the nonexistence of the positive solution. An example is also given to illustrate the main results.

For the sake of convenience, we list the following hypotheses:

(H1) $\lambda>0$ is a parameter, $h:(0, T) \rightarrow[0, \infty)$ is ld-continuous such that $h\left(t_{0}\right)>0$ for at least one $t_{0} \in[0, T)$ and $f:[0, \infty) \rightarrow(0, \infty)$ is continuous;

(H2) $a_{i} \geq 0$ for $i=1, \ldots, m-2,0<\xi_{1}<\xi_{2}<\cdots<\xi_{m-2}<\rho(T)$ and $d=1-\sum_{i=1}^{m-2} a_{i}>0$.

\section{Preliminaries}

Let the Banach space $B=C_{l d}[0, T]$ (see [2]) be endowed with the norm $\|u\|=\sup _{t \in[0, T]}|u(t)|$, and choose the cone $P \subset B$ defined by

$$
P=\left\{\begin{array}{c}
u \in B: u(t) \geq 0 \text { for } t \in[0, T] \text { and } \\
u^{\Delta \nabla}(t) \leq 0, u^{\Delta}(t) \geq 0 \text { for } t \in(0, T), u(0)=0
\end{array}\right\} .
$$

Clearly, by $\sum_{i=1}^{m-2} a_{i}<1$, we can obtain $\|u\|=u(T)$ for $u \in P$. Define the operator $A_{\lambda}: P \rightarrow B$ by

$$
\begin{aligned}
A_{\lambda} u(t)=\int_{0}^{t} \varphi_{q} & \left(-\int_{0}^{s} \lambda a(\tau) f(u(\tau)) \nabla \tau+d \int_{0}^{T} \lambda a(\tau) f(u(\tau)) \nabla \tau\right. \\
& \left.-d \sum_{i=1}^{m-2} a_{i} \int_{0}^{\xi_{i}} \lambda a(\tau) f(u(\tau)) \nabla \tau\right) \Delta s
\end{aligned}
$$

for $0 \leq t \leq T$.

Now we list a lemma which is needed later.

Lemma 2.1. [18, Lemma 2.6] Assume $g: \mathbb{R} \rightarrow \mathbb{R}$ is continuous, $g: \mathbb{T} \rightarrow \mathbb{R}$ is delta differentiable on $\mathbb{T}_{\kappa}$, and $f: \mathbb{R} \rightarrow \mathbb{R}$ is continuous differentiable. Then there exists $c$ in the real interval $[\rho(t), t]$ with

$$
(f \circ g)^{\nabla}(t)=f^{\prime}(g(c)) g^{\nabla}(t) .
$$


By the definition of $A_{\lambda}$, the monotonicity of $\varphi_{q}(x)$ and Lemma 2.1, it is easy to see that for each $u \in P, A_{\lambda} u \in P$ and satisfies (1.2). In addition, since $\left(\varphi_{p}\left(u^{\Delta}(t)\right)\right)^{\nabla}=-\lambda h(t) f(u(t))<0$, and $\varphi_{p}\left(u^{\Delta}(T)\right)=\sum_{i=1}^{m-2} a_{i} \varphi_{p}\left(u^{\Delta}\left(\xi_{i}\right)\right)$, then $A_{\lambda} u(T)$ is the maximum value of $A_{\lambda} u(t)$. Similar as the proof of Lemma 2.5 in [18], we can easily get that $A_{\lambda}: P \rightarrow P$ is completely continuous. And from [18, Lemma 2.6], for $u \in P$, we have $u(t) \geq \frac{t}{T}\|u\|, t \in[0, T]$. Thus, each fixed point of the operator $A_{\lambda}$ in $P$ is a positive solution of $(1.1),(1.2)$.

In order to prove our main result, the following fixed point theorem is crucial.

Lemma $2.2([9,13])$. Let $P$ be a cone in a Banach space $X$. Assume $\Omega_{1}, \Omega_{2}$ are open subsets of $X$ with $0 \in \Omega_{1}, \bar{\Omega}_{1} \subset \Omega_{2}$. If $A: P \cap\left(\bar{\Omega}_{2} \backslash \Omega_{1}\right) \rightarrow P$ is a completely continuous operator such that either

(i) $\|A x\| \leq\|x\|, \forall x \in P \cap \partial \Omega_{1}$ and $\|A x\| \geq\|x\|, \forall x \in P \cap \partial \Omega_{2}$, or

(ii) $\|A x\| \geq\|x\|, \forall x \in P \cap \partial \Omega_{1}$ and $\|A x\| \leq\|x\|, \forall x \in P \cap \partial \Omega_{2}$.

Then $A$ has a fixed point in $P \cap\left(\overline{\Omega_{2}} \backslash \Omega_{1}\right)$.

\section{Characterization of eigenvalues}

In this section, we shall provide conditions under which the set of eigenvalues $E$ contains/is an interval. To begin, we shall define some important constants

$$
\begin{gathered}
C_{1}=\int_{0}^{T} \varphi_{q}\left(d \int_{0}^{T} h(\tau) \nabla \tau\right) \Delta s, \\
C_{2}=\frac{\xi_{m-2}}{T} \int_{\xi_{m-2}}^{T} \varphi_{q}\left(\int_{s}^{T} h(\tau) \nabla \tau\right) \Delta s,
\end{gathered}
$$

and denote

$$
E=\{\lambda>0:(1.1),(1.2) \text { has a positive solution }\} .
$$

Theorem 3.1. Assume (H1), (H2) hold, then there exists $c>0$, such that the interval $(0, c] \subseteq E$.

Proof. Let $r>0$ be given and denote $\bar{P}_{r}=\{u \in P:\|u\| \leq r\}$. Define

$$
c=\varphi_{p}\left(r / C_{1}\right) / M,
$$

where $M=\sup _{u \in[0, r]} f(u), C_{1}$ is defined in (3.1).

Let $\lambda \in(0, c]$, we shall prove that $A_{\lambda}$ maps $\bar{P}_{r}$ into $\bar{P}_{r}$. For $u \in \bar{P}_{r}, A_{\lambda}(u) \in$ $P$. Thus, it remains to check that $\left\|A_{\lambda} u\right\| \leq r$. Using (3.3) we get

$$
\begin{aligned}
\left\|A_{\lambda} u\right\|=\sup _{t \in[0, T]} & {\left[\int _ { 0 } ^ { t } \varphi _ { q } \left(-\int_{0}^{s} \lambda h(\tau) f(u(\tau)) \nabla \tau+d \int_{0}^{T} \lambda h(\tau) f(u(\tau)) \nabla \tau\right.\right.} \\
& \left.\left.-d \sum_{i=1}^{m-2} a_{i} \int_{0}^{\xi_{i}} \lambda h(\tau) f(u(\tau)) \nabla \tau\right) \Delta s\right]
\end{aligned}
$$




$$
\begin{aligned}
& \leq \int_{0}^{T} \varphi_{q}\left(d \int_{0}^{T} \lambda h(\tau) f(u(\tau)) \nabla \tau\right) \Delta s \\
& \leq \varphi_{q}(\lambda M) \int_{0}^{T} \varphi_{q}\left(d \int_{0}^{T} h(\tau) \nabla \tau\right) \Delta s \\
& =\varphi_{q}(\lambda M) C_{1} \leq \varphi_{q}(c M) C_{1}=r .
\end{aligned}
$$

Hence, $A_{\lambda}\left(\bar{P}_{r}\right) \subset \bar{P}_{r}$, Leray-Schauder fixed point theorem guarantee that $A_{\lambda}$ has a fixed point in $\bar{P}_{r}$. Obviously, this fixed point is also a positive solution of (1.1), (1.2), and therefore $\lambda$ is an eigenvalue of (1.1), (1.2). Since $\lambda \in(0, c]$ is arbitrary, it follows that the interval $(0, c] \subseteq E$.

Theorem 3.2. Suppose (H1), (H2) hold, and assume that $f(u)$ is nondecreasing in $(0, \infty)$. If $\lambda_{0} \in E$, then for $\lambda \in\left(0, \lambda_{0}\right)$, we have $\lambda \in E$.

Proof. Suppose that $u_{0}$ is a positive solution for the problem (1.1), (1.2) for $\lambda=\lambda_{0}$. Let

$$
\bar{P}_{u_{0}}=\left\{u \in P: u(t) \leq u_{0}(t), t \in[0, T]\right\}
$$

For any $u \in \bar{P}_{u_{0}}, \lambda \in\left(0, \lambda_{0}\right)$, by (H1), (H2) and the monotonicity of $f$, we have

$$
A_{\lambda} u(t) \leq A_{\lambda_{0}} u_{0}(t)=u_{0}(t) \text { for } t \in[0, T] .
$$

Hence $A_{\lambda}\left(\bar{P}_{u_{0}}\right) \subseteq \bar{P}_{u_{0}}$. By Leray-Schauder fixed point theorem, $A_{\lambda}$ has a fixed point in $\bar{P}_{u_{0}}$, and this fixed point is a positive solution of (1.1), (1.2), thus $\lambda$ is an eigenvalue of (1.1), (1.2).

Theorem 3.3. Suppose $(\mathrm{H} 1)$, ( $\mathrm{H} 2)$, and assume $f(u)$ is nondecreasing in $(0, \infty), \lambda$ is an eigenvalue and $u$ is the corresponding eigenfunction of (1.1), (1.2), then

$$
\frac{\varphi_{p}\left(l / C_{1}\right)}{f(l)} \leq \lambda \leq \frac{\varphi_{p}\left(l / C_{2}\right)}{f\left(l \xi_{m-2} / T\right)}
$$

where $l=\|u\|$.

Proof. Observe that

$$
l=u(T)=A_{\lambda} u(T) \leq \int_{0}^{T} \varphi_{q}\left(d \int_{0}^{T} \lambda h(\tau) f(u(\tau)) \nabla \tau\right) \Delta s \leq \varphi_{q}(\lambda f(l)) C_{1},
$$

hence we have $\lambda \geq \varphi_{p}\left(l / C_{1}\right) / f(l)$. 
Since $u(t) \geq \frac{t}{T}\|u\| \geq \frac{\xi_{m-2} l}{T}, t \in\left[\xi_{m-2}, T\right]$, then in view of the monotonicity of $f$, we have

$$
\begin{gathered}
l \geq u\left(\xi_{m-2}\right)=A_{\lambda} u\left(\xi_{m-2}\right) \geq \frac{\xi_{m-2}}{T} A_{\lambda} u(T) \\
\geq \frac{\xi_{m-2}}{T}\left[\int _ { 0 } ^ { T } \varphi _ { q } \left(-\int_{0}^{s} \lambda h(\tau) f(u(\tau)) \nabla \tau+d \int_{0}^{T} \lambda h(\tau) f(u(\tau)) \nabla \tau\right.\right. \\
\left.\left.\quad-d \sum_{i=1}^{m-2} a_{i} \int_{0}^{T} \lambda h(\tau) f(u(\tau)) \nabla \tau\right) \Delta s\right] \\
=\frac{\xi_{m-2}}{T} \int_{0}^{T} \varphi_{q}\left(\int_{s}^{T} \lambda h(\tau) f(u(\tau)) \nabla \tau\right) \Delta s \\
\geq \frac{\xi_{m-2}}{T} \int_{\xi_{m-2}}^{T} \varphi_{q}\left(\int_{s}^{T} \lambda h(\tau) f(u(\tau)) \nabla \tau\right) \Delta s \\
\geq \varphi_{q}\left(\lambda f\left(\xi_{m-2} l / T\right)\right) C_{2},
\end{gathered}
$$

thus $\lambda \leq \varphi_{p}\left(l / C_{2}\right) / f\left(\xi_{m-2} l / T\right)$.

\section{Eigenvalue intervals}

In this section, we shall apply Lemma 2.2 to derive explicit eigenvalue intervals, and also give sufficient conditions that the problem (1.1), (1.2) has at least one or two positive solutions. For convenience, we introduce

$$
\begin{aligned}
F_{0}=\limsup _{u \rightarrow 0^{+}} \frac{f(u)}{\varphi_{p}(u)}, & f_{0}=\liminf _{u \rightarrow 0^{+}} \frac{f(u)}{\varphi_{p}(u)} \\
F_{\infty}=\limsup _{u \rightarrow+\infty} \frac{f(u)}{\varphi_{p}(u)}, & f_{\infty}=\liminf _{u \rightarrow+\infty} \frac{f(u)}{\varphi_{p}(u)} .
\end{aligned}
$$

Theorem 4.1. Suppose (H1), (H2) hold. Then for each

$$
\left(f_{\infty}\right)^{-1} \varphi_{q}\left(\xi_{m-2} C_{2} / T\right)<\lambda<\left(F_{0}\right)^{-1} \varphi_{q}\left(C_{1}\right),
$$

the boundary value problem (1.1), (1.2) has at least one positive solution. Here we impose $\left(f_{\infty}\right)^{-1} \varphi_{q}\left(\xi_{m-2} C_{2} / T\right)=0$ if $f_{\infty}=\infty$ and $\left(F_{0}\right)^{-1} \varphi_{q}\left(C_{1}\right)=\infty$ if $F_{0}=0$.

Proof. Let $\lambda$ satisfy (4.1) and $\varepsilon>0$ be such that

$$
\left(f_{\infty}-\varepsilon\right)^{-1} \varphi_{q}\left(\xi_{m-2} C_{2} / T\right) \leq \lambda \leq\left(F_{0}+\varepsilon\right)^{-1} \varphi_{q}\left(C_{1}\right) .
$$

By the definition of $F_{0}$, we see that there exists $r_{1}>0$ such that

$$
f(u) \leq\left(F_{0}+\varepsilon\right) \varphi_{p}(u), 0<u \leq r_{1} .
$$


So, if $u \in \partial P_{r_{1}}$, then by (4.3) and (4.2) we have

$$
\begin{aligned}
\left\|A_{\lambda} u\right\|=A_{\lambda} u(T) & \leq \int_{0}^{T} \varphi_{q}\left(d \int_{0}^{T} \lambda h(\tau) f(u(\tau)) \nabla \tau\right) \Delta s \\
& \leq \varphi_{q}\left(\lambda\left(F_{0}+\varepsilon\right)\right) C_{1}\|u\| \leq\|u\| .
\end{aligned}
$$

Hence if we let $\Omega_{1}=\left\{u \in B:\|u\|<r_{1}\right\}$, then

$$
\left\|A_{\lambda} u\right\| \leq\|u\| \text { for } u \in P \cap \partial \Omega_{1} .
$$

Let $r_{3}>0$ be such that

$$
f(u) \geq\left(f_{\infty}-\varepsilon\right) \varphi_{p}(u) \text { for } u \geq r_{3} .
$$

If $u \in B$ with $\|u\|=r_{2}:=\max \left\{2 r_{1}, T r_{3} / \xi_{m-2}\right\}$. Then in view of (4.5) we have

$$
\begin{aligned}
\left\|A_{\lambda} u\right\| & \geq A_{\lambda} u\left(\xi_{m-2}\right) \geq \frac{\xi_{m-2}}{T} A_{\lambda} u(T) \\
& \geq \frac{\xi_{m-2}}{T} \int_{0}^{T} \varphi_{q}\left(\int_{s}^{T} \lambda h(\tau) f(u(\tau)) \nabla \tau\right) \Delta s \\
& \geq \frac{\xi_{m-2}}{T} \int_{\xi_{m-2}}^{T} \varphi_{q}\left(\int_{s}^{T} \lambda h(\tau) f(u(\tau)) \nabla \tau\right) \Delta s \\
& \geq \frac{\xi_{m-2}}{T} \int_{\xi_{m-2}}^{T} \varphi_{q}\left(\int_{s}^{T} \lambda h(\tau)\left(f_{\infty}-\varepsilon\right) \varphi_{p}(u(\tau)) \nabla \tau\right) \Delta s \\
& \geq C_{2} \varphi_{q}\left(\lambda\left(f_{\infty}-\varepsilon\right)\right) \frac{\xi_{m-2}}{T}\|u\| \geq\|u\| .
\end{aligned}
$$

Thus if we set $\Omega_{2}=\left\{u \in B:\|u\|<r_{2}\right\}$, then

$$
\left\|A_{\lambda} u\right\| \geq\|u\| \text { for } u \in P \cap \partial \Omega_{2} .
$$

Now, (4.4), (4.6) and Lemma 2.2 guarantee that $A_{\lambda}$ has a fixed point $u \in$ $P \cap\left(\overline{\Omega_{2}} \backslash \Omega_{1}\right)$ with $r_{1} \leq\|u\| \leq r_{2}$, and clearly $u$ is a positive solution of (1.1), $(1.2)$.

Theorem 4.2. Assume (H1), (H2) hold. Then for each

$$
\left(f_{0}\right)^{-1} \varphi_{q}\left(\xi_{m-2} C_{2} / T\right)<\lambda<\left(F_{\infty}\right)^{-1} \varphi_{q}\left(C_{1}\right),
$$

the problem (1.1), (1.2) has at least one positive solution.

Proof. Let $\lambda$ satisfy (4.7) and $\varepsilon>0$, such that

$$
\left(f_{0}-\varepsilon\right)^{-1} \varphi_{q}\left(\xi_{m-2} C_{2} / T\right) \leq \lambda \leq\left(F_{\infty}+\varepsilon\right)^{-1} \varphi_{q}\left(C_{1}\right) .
$$

From the definition of $f_{0}$, we see that there exists $r_{1}>0$ such that

$$
f(u) \geq\left(f_{0}-\varepsilon\right) \varphi_{p}(u) \text { for } 0<u \leq r_{1} .
$$


Further, if $u \in P$ with $\|u\|=r_{1}$, then $u(t) \geq \frac{\xi_{m-2}}{T}\|u\|, t \in\left[\xi_{m-2}, T\right]$, and similar to the second part of Theorem 4 .1, we can obtain that $\left\|A_{\lambda} u\right\| \geq\|u\|$. Thus, if we let $\Omega_{1}=\left\{u \in B:\|u\|<r_{1}\right\}$, then

$$
\left\|A_{\lambda} u\right\| \geq\|u\| \text { for } u \in P \cap \partial \Omega_{1} .
$$

Next, we may choose $R_{2}>0$ such that

$$
f(u) \leq\left(F_{\infty}+\varepsilon\right) \varphi_{p}(u), u \geq R_{2} .
$$

Here there are two cases to consider, namely, where $f$ is bounded and $f$ is unbounded .

Case 1. Suppose $f$ is bounded, then there exists some $M>0$, such that $f(u) \leq M, u \in(0, \infty)$. We define $r_{3}=\max \left\{2 r_{1}, \varphi_{q}(\lambda M) C_{1}\right\}$, and $u \in P$ be such that $\|u\|=r_{3}$, then

$$
\begin{aligned}
\left\|A_{\lambda} u\right\|=A_{\lambda} u(T) & \leq \int_{0}^{T} \varphi_{q}\left(d \int_{0}^{T} \lambda h(\tau) f(u(\tau)) \nabla \tau\right) \Delta s \\
& \leq \varphi_{q}(\lambda M) C_{1} \leq r_{3}=\|u\| .
\end{aligned}
$$

Hence,

$$
\left\|A_{\lambda} u\right\| \leq\|u\| \text { for } u \in \partial P_{r_{3}} .
$$

Case 2. Suppose $f$ is unbounded, then there exists $r_{4}>\max \left\{2 r_{1}, R_{2}\right\}$ such that

$$
f(u) \leq f\left(r_{4}\right), 0<u \leq r_{4} .
$$

Let $u \in P$ be such that $\|u\|=r_{4}$, then by (4.8), we have

$$
\begin{aligned}
\left\|A_{\lambda} u\right\| & =A_{\lambda} u(T) \leq \int_{0}^{T} \varphi_{q}\left(d \int_{0}^{T} \lambda h(\tau) f(u(\tau)) \nabla \tau\right) \Delta s \\
& \leq \varphi_{q}\left(\lambda\left(F_{\infty}+\varepsilon\right)\right) C_{1}\|u\| \leq\|u\| .
\end{aligned}
$$

Thus, (4.11) is also true.

In both Case 1 and Case 2, if we set $\Omega_{2}=\left\{u \in B:\|u\|<r_{2}=\max \left\{r_{3}, r_{4}\right\}\right\}$, then (4.10) hold for $u \in P \bigcap \partial \Omega_{2}$.

Now that we have obtained (4.9) and (4.11), it follows from Lemma 2.2 that $A_{\lambda}$ has a fixed point $u \in P \cap\left(\overline{\Omega_{2}} \backslash \Omega_{1}\right)$, and $r_{1} \leq\|u\| \leq r_{2}$. It is clear that $u$ is a positive solution of (1.1), (1.2).

In the rest of this section, we consider the existence of two positive solutions of $(1.1),(1.2)$. First, we give a lemma.

Lemma 4.1. Suppose (H1), (H2) hold. In addition, assume there exist $r_{2}>$ $r_{1}>0$, such that

$$
\begin{gathered}
\max _{0 \leq u \leq r_{1}} f(u) \leq \varphi_{p}\left(r_{1} / C_{1}\right) / \lambda, \\
\min _{\xi_{m-2} r_{2} / T \leq u \leq r_{2}} f(u) \geq \varphi_{p}\left(r_{2} / C_{2}\right) / \lambda .
\end{gathered}
$$


Then, (1.1), (1.2) has a solution $u \in P$ with $r_{1} \leq\|u\| \leq r_{2}$.

Proof. The proof of Lemma 4.3 is similar to that of Theorem 4.2, we omit it here.

For the remainder of the paper, we will need the following condition:

(H3) $\sup _{r>0} \min _{u \in\left(\xi_{m-2} r / T, r\right)} f(u)>0$.

Let

$$
\begin{gathered}
\lambda_{1}=\sup _{r>0} \frac{\varphi_{p}\left(r / C_{1}\right)}{\max _{0 \leq u \leq r} f(u)}, \\
\lambda_{2}=\inf _{r>0} \frac{\varphi_{p}\left(r / C_{2}\right)}{\min _{\xi_{m-2} r / T \leq u \leq r} f(u)} .
\end{gathered}
$$

In view of (H1) and (H3), we can easily obtain that $0<\lambda_{1} \leq \infty$ and $0 \leq \lambda_{2}<$ $\infty$.

Theorem 4.3. Suppose (H1)-(H3) hold, if $f_{0}=\infty$ and $f_{\infty}=\infty$, then the problem (1.1), (1.2) has at least two positive solutions for each $\lambda \in\left(0, \lambda_{1}\right)$.

Proof. Define

$$
a(r)=\frac{\varphi_{p}\left(r / C_{1}\right)}{\max _{0 \leq u \leq r} f(u)}, \quad r>0
$$

then by $(\mathrm{H} 1), f_{0}=\infty$ and $f_{\infty}=\infty$, we have that $a(r):(0, \infty) \rightarrow(0, \infty)$ is continuous and

$$
\lim _{r \rightarrow 0} a(r)=\lim _{r \rightarrow \infty} a(r)=0 .
$$

By (4.15), there exists $r_{0} \in(0, \infty)$, such that $a\left(r_{0}\right)=\sup _{r>0} a(r)=\lambda_{1}$, then for $\lambda \in\left(0, \lambda_{1}\right)$, there exist constants $c_{1}, c_{2}\left(0<c_{1}<r_{0}<c_{2}<\infty\right)$ with $a\left(c_{1}\right)=a\left(c_{2}\right)=\lambda$. Thus

$$
\begin{aligned}
& f(u) \leq \varphi_{p}\left(c_{1} / C_{1}\right) / \lambda \text { for } u \in\left[0, c_{1}\right], \\
& f(u) \leq \varphi_{p}\left(c_{2} / C_{1}\right) / \lambda \text { for } u \in\left[0, c_{2}\right] .
\end{aligned}
$$

On the other hand, applying the conditions $f_{0}=\infty$ and $f_{\infty}=\infty$, there exist constants $d_{1}, d_{2}\left(0<d_{1}<c_{1}<r_{0}<c_{2}<d_{2}<\infty\right)$, with

$$
f(u) / \varphi_{p}(u) \geq \lambda^{-1} \varphi_{q}\left(\xi_{m-2} C_{2} / T\right) \text { for } u \in\left(0, d_{1}\right) \cup\left(\xi_{m-2} d_{2} / T, \infty\right),
$$

$$
\begin{array}{r}
\min _{\xi_{m-2} d_{1} / T \leq u \leq d_{1}} f(u) \geq \lambda^{-1} \varphi_{p}\left(d_{1} / C_{2}\right), \\
\min _{\xi_{m-2} d_{2} / T \leq u \leq d_{2}} f(u) \geq \lambda^{-1} \varphi_{p}\left(d_{2} / C_{2}\right) .
\end{array}
$$

By (4.17) and (4.20), (4.18) and (4.21), Lemma 4.3, we can complete the proof.

Theorem 4.4. Suppose (H1)-(H3) hold, if $f_{0}=0$ and $f_{\infty}=0$, then for each $\lambda \in\left(\lambda_{2}, \infty\right)$ the problem (1.1), (1.2) has at least two positive solutions. 
Proof. Define

$$
b(r)=\frac{\varphi_{p}\left(r / C_{2}\right)}{\min _{\xi_{m-2} r / T \leq u \leq r} f(u)}, \quad r \in(0, \infty) .
$$

By $f_{0}=0$ and $f_{\infty}=0$ we easily see that $b(r):(0, \infty) \rightarrow(0, \infty)$ is continuous and

$$
\lim _{r \rightarrow 0} b(r)=\lim _{r \rightarrow \infty} b(r)=\infty .
$$

Thus there exists $r_{0} \in(0, \infty)$, such that $b\left(r_{0}\right)=\inf _{r>0} b(r)=\lambda_{2}$. For $\lambda \in$ $\left(\lambda_{2}, \infty\right)$, there exist constants $d_{1}, d_{2}\left(0<d_{1}<r_{0}<d_{2}<\infty\right)$ with $b\left(d_{1}\right)=$ $b\left(d_{2}\right)=\lambda$.

Therefore

$$
\begin{aligned}
& f(u) \geq \varphi_{p}\left(d_{1} / C_{2}\right) / \lambda \text { for } u \in\left[\xi_{m-2} d_{1} / T, d_{1}\right], \\
& f(u) \geq \varphi_{p}\left(d_{2} / C_{2}\right) / \lambda \text { for } u \in\left[\xi_{m-2} d_{2} / T, d_{2}\right] .
\end{aligned}
$$

On the other hand, using $f_{0}=0$, we know that there is a constant $c_{1}\left(0<c_{1}<\right.$ $\left.d_{1}\right)$ with

$$
\begin{gathered}
f(u) / \varphi_{p}(u) \leq \lambda^{-1} \varphi_{q}\left(C_{1}\right) \text { for } u \in\left(0, c_{1}\right) . \\
\max _{0 \leq u \leq c_{1}} f(u) \leq \varphi_{p}\left(c_{1} / C_{1}\right) / \lambda .
\end{gathered}
$$

In view of $f_{\infty}=0$, there exists a constant $c_{2} \in\left(d_{2}, \infty\right)$ such that

$$
f(u) / \varphi_{p}(u) \leq \varphi_{q}\left(C_{1} \lambda\right) \text { for } u \in\left(c_{2}, \infty\right) .
$$

Let $M=\sup _{u \in\left[0, c_{2}\right]} f(u)$ and $c_{2} \geq C_{1} \varphi_{q}(\lambda M)$. It is easily seen that

$$
\max _{0 \leq u \leq c_{2}} f(u) \leq \varphi_{p}\left(c_{2} / C_{1}\right) / \lambda .
$$

By (4.22), (4.23) and Lemma 4.3, we can complete the proof.

\section{Nonexistence}

In this section, we give some sufficient conditions for the nonexistence of positive solution to the problem (1.1), (1.2).

Theorem 5.1. Suppose (H1)-(H3) hold, if $F_{0}<\infty, F_{\infty}<\infty$, then there exists a $\lambda_{0}>0$ such that for all $0<\lambda<\lambda_{0}$, (1.1), (1.2) has no positive solution.

Proof. Since $F_{0}<\infty$ and $F_{\infty}<\infty$, there exist positive numbers $l_{1}, l_{2}, r_{1}$ and $r_{2}$ such that $r_{1}<r_{2}$ and

$$
\begin{aligned}
& f(u) \leq l_{1} \varphi_{p}(u) \text { for } u \in\left[0, r_{1}\right], \\
& f(u) \leq l_{2} \varphi_{p}(u) \text { for } u \in\left[r_{2}, \infty\right) .
\end{aligned}
$$

Let $L=\max \left\{l_{1}, l_{2}, \max _{r_{1} \leq u \leq r_{2}}\left\{f(u) \varphi_{q}(u\}\right\}\right.$, then we have

$$
f(u) \leq L \varphi_{p}(u) \text { for } u \in[0, \infty) .
$$


Assume $v(t)$ is a positive solution of (1.1), (1.2). We will show that this leads to a contradiction for $0<\lambda<\lambda_{0}:=L^{-1} \varphi_{q}\left(C_{1}\right)$. Since $A_{\lambda} v(t)=v(t)$ for $t \in[0, T]$, then

$$
\begin{aligned}
\|v\| & =\left\|A_{\lambda} v\right\| \leq \int_{0}^{T} \varphi_{q}\left(d \int_{0}^{T} \lambda h(\tau) f(v(\tau)) \nabla \tau\right) \Delta s \\
& \leq \varphi_{q}(\lambda L)\|v\| \int_{0}^{T} \varphi_{q}\left(d \int_{0}^{T} h(\tau) \nabla \tau\right) \Delta s=\varphi_{q}(\lambda L)\|v\| C_{1}<\|v\|,
\end{aligned}
$$

which is a contradiction. Therefore, (1.1), (1.2) has no positive solution.

Theorem 5.2. Suppose (H1)-(H3) hold, if $f_{0}>0, f_{\infty}>0$, then there exist a $\lambda_{0}>0$ such that for all $\lambda>\lambda_{0},(1.1),(1.2)$ has no positive solution.

Proof. By $f_{0}>0, f_{\infty}>0$, we know that there exist $m_{1}, m_{2}, r_{1}$ and $r_{2}$ such that $r_{1}<r_{2}$ and

$$
\begin{aligned}
& f(u) \geq m_{1} \varphi_{p}(u) \text { for } u \in\left[0, r_{1}\right], \\
& f(u) \geq m_{2} \varphi_{p}(u) \text { for } u \in\left[r_{2}, \infty\right) .
\end{aligned}
$$

Let $m_{3}=\min \left\{m_{1}, m_{2}, \min _{r_{1} \leq u \leq r_{2}}\left\{f(u) \varphi_{q}(u)\right\}\right\}>0$, then we get

$$
f(u) \geq m_{3} \varphi_{p}(u) \text { for } u \in[0, \infty) .
$$

Assume $v(t)$ is a positive solution of (1.1), (1.2). We will show that this leads to a contradiction for $\lambda>\lambda_{0}:=\left(m_{3}\right)^{-1} \varphi_{p}\left(\xi_{m-2} C_{2} / T\right)$. Since $A_{\lambda} v(t)=v(t)$ for $t \in[0, T]$, then

$$
\begin{aligned}
\|v\| & =\left\|A_{\lambda} v\right\| \\
& \geq \frac{\xi_{m-2}}{T} A_{\lambda} v(T) \geq \frac{\xi_{m-2}}{T} \int_{\xi_{m-2}}^{T} \varphi_{q}\left(\int_{s}^{T} \lambda h(\tau) f(v(\tau)) \nabla \tau\right) \Delta s \\
& =\varphi_{q}\left(\lambda m_{3}\right) \frac{\xi_{m-2}}{T}\|v\| C_{2}>\varphi_{q}\left(\lambda_{0} m_{3}\right) \frac{\xi_{m-2}}{T}\|v\| C_{2}=\|v\|,
\end{aligned}
$$

which is a contradiction. Thus, (1.1), (1.2) has no positive solution.

Example 5.3. Let $\mathbb{T}=\left\{1-\left(\frac{1}{2}\right)^{\mathbb{N}_{0}}\right\} \cup\{1\}$, where $\mathbb{N}_{0}$ denotes the set of all nonnegative integers. Taking $T=1, p=2, m=3, a_{1}=1 / 4, \xi_{1}=1 / 2$, if we let $h(s)=1$, then $C_{1}=\int_{0}^{1} \frac{4}{3} \int_{0}^{1} 1 \nabla \tau \Delta s=\frac{4}{3}, C_{2}=\frac{1}{2} \int_{1 / 2}^{1} \int_{s}^{1} 1 \nabla \tau \Delta s=1 / 3$. Suppose

$$
f(u)=\frac{1+150 u}{1+u} u\left(\frac{3}{2}+\sin u\right), u \geq 0 .
$$

Clearly $f$ is always increasing and $F_{0}=f_{0}=3 / 2, F_{\infty}=375, f_{\infty}=75$.

By direct calculation, it is easy to get that

$$
\begin{gathered}
\left(f_{\infty}\right)^{-1} \varphi_{q}\left(\xi_{1} C_{2} / T\right)=0.08, \quad\left(F_{0}\right)^{-1} \varphi_{q}\left(C_{1}\right)=0.5 \\
l^{-1} \varphi_{q}\left(C_{1}\right)=0.0133 \text { and }\left(m_{3}\right)^{-1} \varphi_{p}\left(\xi_{1} C_{2} / T\right)=4 .
\end{gathered}
$$


Thus the boundary value problem

$$
\begin{gathered}
u^{\Delta \nabla}(t)+f(u(t))=0, \\
u(0)=0, u^{\Delta}(1)=3 / 4 u^{\Delta}(1 / 2)
\end{gathered}
$$

has at least one positive solutions for $0.08<\lambda<0.5$, has no solution in $P$ for $0<\lambda<0.0013$ or $\lambda>4$ by Theorem 4.1, Theorem 5.1 and Theorem 5.2 respectively.

\section{References}

[1] R. P. Agarwal, H. Lü, and D. O'Regan, Eigenvalues and the one-dimensional pLaplacian, J. Math. Anal. Appl. 266 (2002), no. 2, 383-400.

[2] D. R. Anderson, Solutions to second-order three-point problems on time scales, J. Difference Equ. Appl. 8 (2002), no. 8, 673-688.

[3] D. R. Anderson, R. Avery, and J. Henderson, Existence of solutions for a one dimensional p-Laplacian on time-scales, J. Difference Equ. Appl. 10 (2004), no. 10, 889-896.

[4] F. M. Atici, D. C. Biles, and A. Lebedinsky, An application of time scales to economics, Math. Comput. Modelling 43 (2006), no. 7-8, 718-726.

[5] M. Bohner and A. Peterson, Dynamic Equations on Time Scales: An Introduction with Applications, Birkhauser, Boston, 2001.

[6] _ Advances in Dynamic Equations on Time Scales, Birkhäuser, Boston, 2003.

[7] J. J. DaCunha, J. M. Davis, and P. K. Singh, Existence results for singular three point boundary value problems on time scales, J. Math. Anal. Appl. 295 (2004), no. 2, 378391.

[8] L. Erbe, A. Peterson, and R. Mathsen, Existence, multiplicity, and nonexistence of positive solutions to a differential equation on a measure chain, J. Comput. Appl. Math. 113 (2000), no. 1-2, 365-380.

[9] D. Guo and V. Lakshmikantham, Nonlinear Problems in Abstract Cones, Academic press, San Diego, 1988.

[10] S. Hilger, Analysis on measure chains - a unified approach to continuous and discrete calculus, Results Math. 18 (1990), no. 1-2, 18-56.

[11] M. A. Jones, B. Song, and D. M. Thomas, Controlling wound healing through debridement, Math. Comput. Modelling 40 (2004), no. 9-10, 1057-1064.

[12] E. R. Kaufmann and Y. N. Raffoul, Eigenvalue problems for a three-point boundaryvalue problem on a time scale, Electron. J. Qual. Theory Differ. Equ. 2004 (2004), no. $2,10 \mathrm{pp}$.

[13] M. Krasnoselskii, Positive Solutions of Operator Equations, P. Noordhoff Ltd. Groningen, 1964.

[14] V. Spedding, Taming nature's numbers, New Scientist 179 (2003), no. 2404, 28-31.

[15] H. R. Sun and W. T. Li, Positive solutions for nonlinear three-point boundary value problems on time scales, J. Math. Anal. Appl. 299 (2004), no. 2, 508-524.

[16] _ Positive solutions for nonlinear $m$-point boundary value problems on time scales, Acta Math. Sinica (Chin. Ser.) 49 (2006), no. 2, 369-380.

$[17]$ _ Positive solutions for $p$-Laplacian $m$-point boundary value problems on time scales, Tainwanese J. Math. 12 (2008), no. 1, 93-115.

[18] _ Multiple positive solutions for p-Laplacian m-point boundary value problems on time scales, Appl. Math. Comput. 182 (2006), no. 1, 478-491.

[19] D. M. Thomas, L. Vandemuelebroeke, and K. Yamaguchi, A mathematical evolution model for phytoremediation of metals, Discrete Contin. Dyn. Syst. Ser. B 5 (2005), no. $2,411-422$. 


\section{Mingzhou Guo}

School of Mathematics and Statistics

LANZHOU UNIVERSITY

Lanzhou, Gansu 730000, P. R. China

E-mail address: guo.math@163.com

HONG-Rui Sun

School of Mathematics and Statistics

LANZHOU UNIVERSITY

Lanzhou, Gansu 730000, P. R. China

E-mail address: hrsun@lzu.edu.cn 